\title{
Flavonoid-rich berry-extract treatment decreases glucose uptake in human intestinal Caco-2 cells
}

\author{
F. Alzaid, H. M. Cheung, P. A. Sharp, V. R. Preedy and H. Wiseman \\ Diabetes and Nutritional Sciences Division, King's College London, Franklin-Wilkins Building, \\ 150 Stamford Street, London SE1 9NH, UK
}

Berries are a rich dietary source of bioactive polyphenols, including flavonoids, such as anthocyanins ${ }^{(1)}$. Dietary flavonoids are known to decrease the expression of the facilitative GLUT2 and the sodium-glucose cotransporter (SGLT1) genes, the two main glucose transporters in human subjects' intestinal Caco-2 cells ${ }^{(2)}$. Acute inhibitory effects on glucose absorption have previously been observed in the presence of flavonoids, however, little is known regarding genomic effects on uptake ${ }^{(3)}$. The present study investigated the influence of a flavonoid-rich berry-extract on glucose uptake in human subject's intestinal Caco-2 cell monolayers.

Human subject's intestinal Caco-2 cells, cultured for $19 \mathrm{~d}$, were treated, acutely $(15 \mathrm{~min})$ or chronically $(16 \mathrm{~h})$ with a flavonoid-rich berry-extract (OptiBerry; InterHealth Nutraceuticals, Benicia, CA, USA) at a final concentration of $0.125 \%$ (w/v) then subjected to radiolabelled glucose for 2 min, in the presence or absence of Na. Uptake was determined by liquid scintillation counting of cell lysate. Data were normalised to cell protein concentration and presented as uptake as a percentage of control. Statistical significance was determined by Student's $t$-test $(P \leq 0.05 ; n 12)$.

Results indicated that berry flavonoids significantly decrease glucose uptake in human subject's intestinal Caco-2 cells both acutely and chronically. These inhibitory effects are evident in both $\mathrm{Na}$-dependent and $\mathrm{Na}$-independent glucose uptake pathways. Studies are in progress to investigate the biological relevance of the observed effects in relation to berry consumption and glucose metabolism. Potentially, such berry-extracts may be useful in the dietary modulation of postprandial glucose homeostasis.
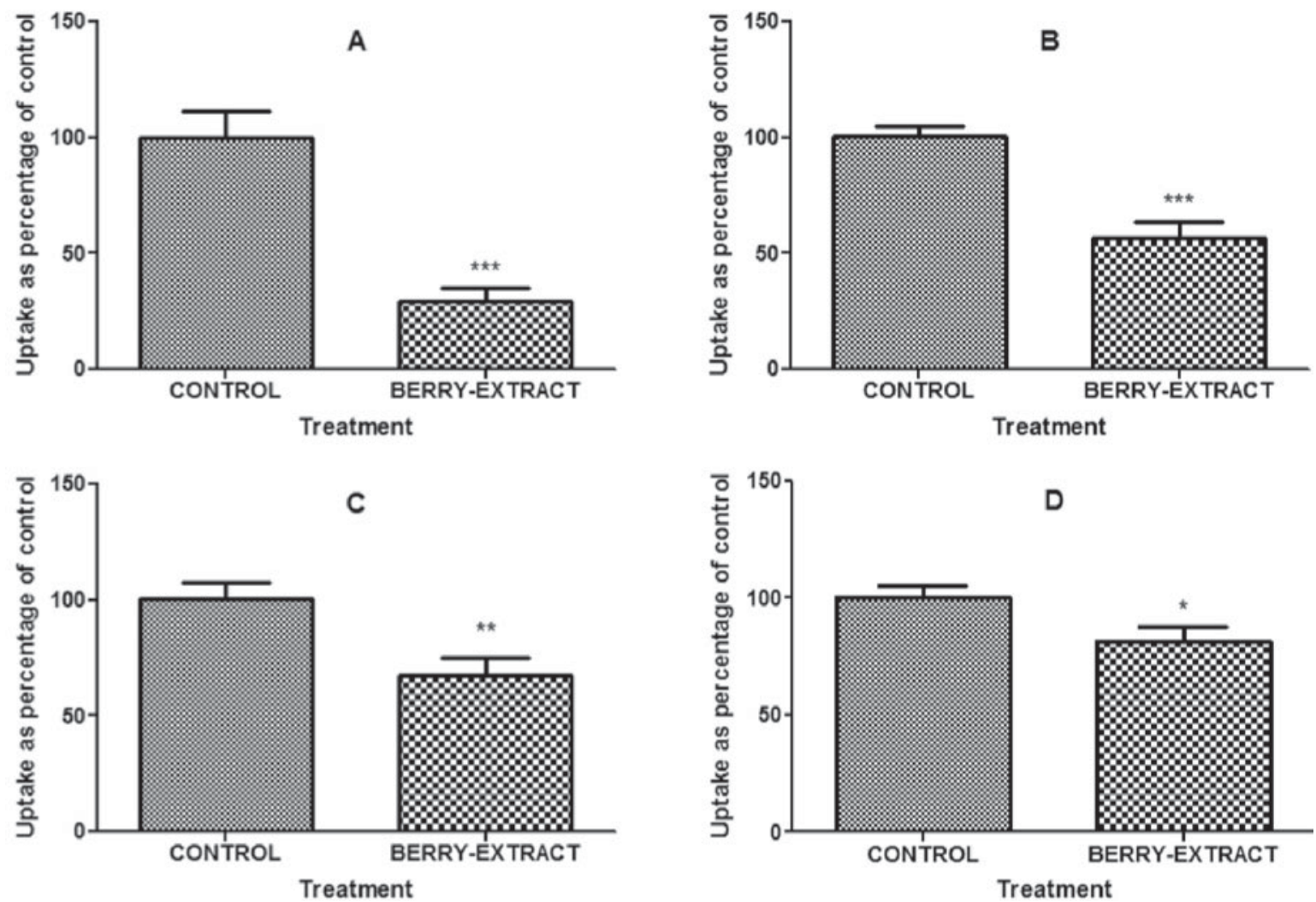

Fig. 1. Effects of berry-extract treatment on $\mathrm{Na}$-dependent and -independent glucose uptake in human subject's intestinal Caco-2 cells. A. Acute berry-extract treatment in the presence of $\mathrm{Na}$. B. Acute berry-extract treatment in the absence of Na. C. Chronic berry-extract treatment in the presence of Na. D. Chronic berry-extract treatment in the absence of Na. Data expressed as mean uptake as a percentage of control (SEM), $n 12 ; *: P \leq 0.05, * *: P \leq 0.01,,^{* * *}: P \leq 0.001$.

1. Zafra-Stone S, Yasmin T, Bagchi M et al. (2007) Berry anthocyanins as novel antioxidants in human health and disease prevention. Mol Nutr Food Res 51, 675-683.

2. Alzaid F, Pourvali K, Sharp PA et al. (2010) Anthocyanin-rich berry-extract treatment decreases expression of dietary glucose transporter genes in human intestinal Caco-2 cells. Proc. Nut. Soc. 69(OCE4), E326.

3. Johnston K, Sharp P, Clifford M et al. (2005) Dietary polyphenols decrease glucose uptake by human intestinal Caco-2 cells. FEBS Lett 579, $1653-1657$. 